\title{
Identification of structural defects using a simplified algorithm of thermal tomography
}

by M. Bajorek*, A. Nowakowski*

\author{
*Department of Biomedical Engineering, Gdansk University of Technology, Poland, \\ marcin.bajorek@wp.pl, antnowak@biomed.eti.pg.gda.pl
}

\begin{abstract}
This paper concerns modification of the thermal tomography reconstruction algorithm to improve the speed of calculations for faster reconstruction of thermal and geometrical parameters inside a tested structure. We propose to solve the inverse problem in the two step procedure. First, initial rough reconstruction of a tested structure based on the one dimensional (1-D) thermal model. Second, execution of the full 3-D reconstruction procedure, with the first step result as the initial condition for further calculations. To verify value of the proposed method some simulation results of the phantom structure developed for testing the method as well as real reconstruction of this phantom are presented.
\end{abstract}

\section{Introduction}

Probably Vavilov used for the first time the term "thermal tomography" (TT) in [1], though the proposed procedure of determination of internal structure of a tested object was not really "tomographic". Recently he discussed the state of the art in TT, terminology and application for testing of composite materials in [2]. We for several years are studying possibility of using TT in medical diagnostics [3].

Under the term Thermal Tomography we understand reconstruction and visualization of internal structure of thermal properties of a 3-D object, based on solving the inverse problem of heat flow, forced by thermal excitation. The tested structure internally may be unknown, what means that both geometry and thermal parameters are unknown. After forced excitation of a tested object one may use IR-imaging for registration of its surface temperature changes in time. In TT determination of the structure of the object is performed by analysis of temperature transients following any known excitation, in comparison with simulation results of the tested object thermal model, to be modified till the result is the same. The reconstruction is made based on measurements of dynamic thermal processes at the surface of the object. Reconstructed parameters are thermal capacity and thermal conductivity. The advantage of TT over conventional IR thermal imaging is the ability to specify the location of existing structure changes (defects) in the full geometry of the object. Although the concept of this method has been used in several studies, there is still no effective and rapid method to solve the thermal tomography algorithm, due to the complex and time-consuming computational algorithms.

\section{Methodology}

In order to reduce the time of identification of thermal parameters several steps affecting the reconstruction procedure are proposed. The main idea is to introduce in the first phase of the reconstruction a one-dimensional heat transfer model, what means modelling of temperature flow into the structure. The concept is based on extracting as much as possible information about the properties of an object from one-dimensional simulations: detection of areas where there are defects, an initial indication of the depth at which there are defects and determine their approximate size.

In the first stage the simplified procedure of reconstruction algorithm, the measurement thermographic sequence is loaded, then for each pixel or group of pixels procedure for the preparation of results is performed. At the same time the simulation procedure is carried out which takes into account the one-dimensional heat flow model of heat exchange. The following input data to the simulation should be given, among others: excitation parameters, temperature distribution at the surface in the steady state, the thickness of defect and, if known, the approximate thermal parameters of structure and of defects. On the basis of these parameters, the algorithm automatically creates all the possible configurations of defect sizes and localizations and then performs simulation. Number of points for which the 1-D model is calculated depends on the supplied input data. In the last step the measurement results and simulation data are compared. After solution of the inverse problem we expect as results defect location in the structure and identification of defect thermal parameters. The result of reconstruction obtained in the initial phase is passed to the full procedure of thermal tomography, where simulations are performed taking into account the 3-D heat transfer model.

\section{Results}

In order to analyze the properties of the simplified reconstruction algorithm, based on temperature values recorded during ADT testing, series of simulations with defined various terms of defect distribution were conducted. It was assumed that inside the structure with known thermal parameters 9 defects are located at different depths. Figure 1 
shows the graphical result of the reconstruction of the depth of defect's placement. The presented reconstruction results are in good agreement with the location and placement depths of defects.

Visual analysis of presented results shows that the location of defects has been properly carried out. One can see, particularly in Figure 1, the exact shape of all nine defects.

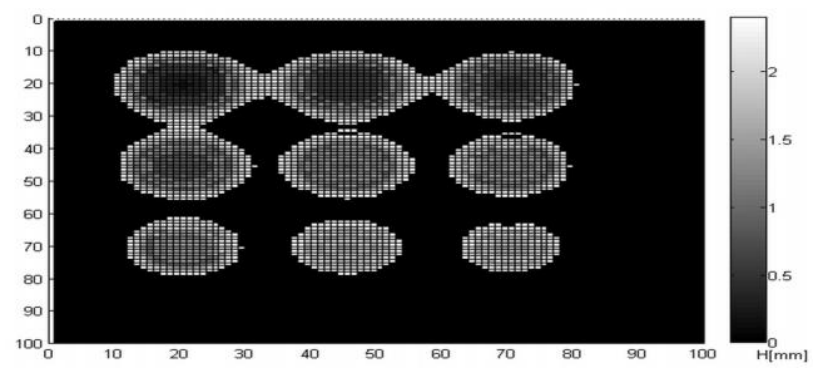

Fig. 1. Reconstruction of the depth of defects

Also correct results of reconstruction of a physical phantom, the actual structure with defects are detected. In the study six defects located at the depth of 2 to $4 \mathrm{~mm}$ were placed. The reconstruction algorithm correctly detected and defined the depth of five defects. Reconstructed depth placement of the defects is shown in Fig 2 . The smallest defect is missing.

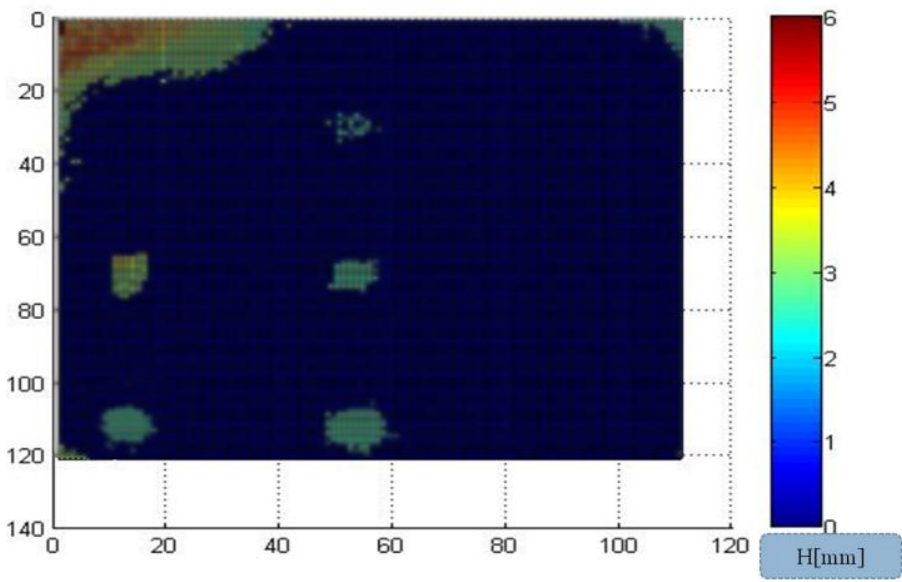

Fig. 2. Absolute error of the depth reconstruction of defects placement for experimental results

If thermal properties of a defect are known the time of reconstruction of a defect's depth is approximately one minute. If the exact value of thermal parameters is not known (only their scope), the reconstruction time which includes detection of defect thermal parameters and its depth is increased to several minutes.

\section{Conclusion}

The presented procedure of solving the thermal inverse problem by two-step reconstruction of a tested structure allows speeding up calculations and creates opportunity for reducing significantly the time of simulations as well as the time of identification of parameters of real structures, maintaining reasonable accuracy of the reconstruction. Use of the 1-D heat exchange model in identification defect procedure can pre-determine the geometrical and thermal parameters of a tested object. Proper interpretation of data obtained in this way allows to significantly simplifying the procedure of following 3-D reconstruction.

\section{REFERENCES}

[1] Vavilov V., Shirayev V., Thermal Tomograph, USSR Patent no. 1.266.308", 1985

[2] Vavilov, V., et al., Thermal (Infrared) Tomography: Terminology, Principal Procedures, and Application to Nondestructive Testing of Composite Materials, Russian Journal of Nondestructive Testing, Vol. 46(3), 151-161, 2010.

[3] Nowakowski A., Quantitative Active Dynamic Thermal IR-Imaging and Thermal Tomography in Medical Diagnostics, The Biomedical Engineering Handbook, Third Edition, Medical Devices and Systems, ed. J. B. Bronzino, CRC Taylor \& Francis, III Infrared Imaging, 22, 22-1 - 22-30, 2006. 